\title{
THE RANGE OF A MULTIMEASURE
}

\author{
LE VAN TU
}

(Received 4 March 1977; revised 2 March 1978)

Communicated by E. Strzelecki

\begin{abstract}
In this paper, the author generalizes the concept of thinness introduced by Kingman and Robertson (1968) to study the convexity of the range of a multimeasure. It is proved that every thin multimeasure taking values in a Fréchet space has convex range, and that, for a suitable measurable space, if a multimeasure is non-atomic, then the weak closure of its range is convex.
\end{abstract}

Subject classification (Amer. Math. Soc. (MOS) 1970): primary 28 A 25, 28 A 45, 46 G 99, $54 \mathrm{C} 60$; secondary 46 A $05,46 \mathrm{G} 10$.

\section{Introduction}

Let $(S, \mathscr{M})$ be a measurable space (that is, a set $S$ in which is defined a $\sigma$-algebra $\mathscr{M}$ of subsets) and let $X$ be a locally convex space. A map $M$ from $\mathscr{M}$ to the family of all non-empty subsets of $X$ is called a multimeasure (or set-valued measure) from $\mathscr{M}$ to $X$ if for every sequence of disjoint sets $A_{i} \in \mathscr{M}(i=1,2, \ldots)$ with $\bigcup_{i=1}^{\infty} A_{i}=A$, the series $\sum_{i=1}^{\infty} M\left(A_{i}\right)$ converges (in the sense of Kluvánek and Knowles (1975), p. 3) to $M(A)$.

The purpose of this paper is to study the range of a multimeasure $M$, that is, the set

$$
R(M)=\bigcup\{M(A): A \in \mathscr{M}\} .
$$

For the case of point-valued measures, Lyapunov (1940) has proved that the range of a measure $m$ from $\mathscr{M}$ to $\mathbf{R}^{n}$ is convex (and compact) if $m$ is non-atomic. This result has been generalized by Kingman and Robertson (1968) and by Knowles (1975) for measures taking values in a locally convex space. In this paper we shall generalize the concept of "thinness" introduced by Kingman and Robertson (1968), p. 348, and apply it to observe the convexity of the range of a multimeasure. 
Our main results are Theorem 2 and Theorem 3. The former states that every thin multimeasure taking values in a Fréchet space has convex range; in the latter we prove that if $\mathscr{M}$ is $M$-essentially countably generated and if $M$ is non-atomic, then the weak closure of $R(M)$ is convex. These results will be presented in Section 3. Section 2 contains definitions and preliminary results which we shall need in Section 3.

I should like to express my gratitude to Professor A. P. Robertson for suggesting this problem and for many helpful discussions. I am also grateful to the referee for his helpful suggestions leading to the presentation of this paper.

\section{Definitions and preliminaries}

Henceforth $(S, \mathscr{M})$ is a measurable space, $X$ is a Hausdorff locally convex space with (topological) dual $X^{\prime}$ and $M$ is a multimeasure from $\mathscr{M}$ to $X$, except where otherwise specified.

A (point-valued) measure $m$ from $\mathscr{M}$ to $X$ is called a selector for $M$ if $m(A) \in M(A)$ for every $A \in \mathscr{H}$. We denote by $\mathscr{S}(M)$ the set of all selector measures of $M$.

Definition 1. A multimeasure $M$ is said to be perfect if

$$
M(A)=\{m(A): m \in \mathscr{S}(M)\}
$$

for every $A \in \mathscr{K}$.

It is clear that if $M$ is a perfect multimeasure, then for every sequence of measures $m_{i} \in \mathscr{S}(M)(i=1,2, \ldots)$ and every sequence of disjoint sets $A_{i} \in \mathscr{M}(i=1,2, \ldots)$ with $\bigcup_{i=1}^{\infty} A_{i}=A$; there exists an $m \in \mathscr{S}(M)$ such that $\sum_{i=1}^{\infty} m_{i}\left(A_{i}\right)=m(A)$. Conversely we have the following lemma the proof of which is trivial.

LEMMA 1. Let $\mathscr{H}$ be a family of measures from $\mathscr{M}$ to $X$ with the property that for every sequence of measures $m_{i} \in \mathscr{H}(i=1,2, \ldots)$ and every sequence of disjoint sets $A_{i} \in \mathscr{M}(i=1,2, \ldots)$ with $\bigcup_{i=1}^{\circ} A_{i}=A$, there exists an $m \in \mathscr{H}$ such that the series $\sum_{i=1}^{\infty} m_{i}\left(A_{i}\right)$ converges to $m(A)$. Then the set-valued map $M: \mathscr{H} \rightarrow X$ defined by $M(E)=\{m(E): m \in \mathscr{H}\}$, for every $E \in \mathscr{M}$, is a perfect multimeasure.

For other results on the perfectness of a multimeasure, we refer to Costé (1975, 1976) and Tolstonogov (1975).

Now let us introduce the concept of atomicity for a multimeasure. Recall that a set $A \in \mathscr{H}$ is called an atom of $\mathscr{K}$ if $A \neq \varnothing$ and $E \in \mathscr{M}, E \subset A$ imply $E=\varnothing$ or $E=A$. 
Definition 2. A set $A \in \mathscr{M}$ is called an atom of $M$ if $M(A) \neq\{0\}$ and $E \in \mathscr{M}, E \subset A$ imply $M(E)=\{0\}$ or $M(A \backslash E)=\{0\}$. The multimeasure $M$ is said to be non-atomic if it has no atoms.

Clearly if $A$ is an atom of $\mathscr{M}$ and if $M(A) \neq\{0\}$, then $A$ is an atom of $M$. Also, if $A$ is an atom of $M$, then it is an atom for any $m \in \mathscr{S}(M)$ with $m(A) \neq 0$.

For convenience of terminology we say that a family $\mathscr{H}$ of measures fills out $M$ if $M(A)=\{m(A): m \in \mathscr{H}\}$ for every $A \in \mathscr{H}$. The following lemma is immediate.

LeMMA 2. Let $M: \mathscr{M} \rightarrow X$ be a perfect multimeasure. If $\mathscr{S}(M)$ contains a subset $\mathscr{H}$ consisting of non-atomic measures such that $\mathscr{H}$ fills out $M$, then $M$ is non-atomic.

A set $E \in \mathscr{M}$ is said to be $M$-null if $M(E \cap F)=\{0\}$ for every $F \in \mathscr{M}$. Now let $\mathscr{M}_{0}$ be a sub- $\sigma$-algebra of $\mathscr{M}$, that is, $\mathscr{M}_{0} \subset \mathscr{M}$. We say that $\mathscr{M}$ is $M$-essentially equal to $\mathscr{M}_{0}$ if for each $A \in \mathscr{M}$, there exists $B \in \mathscr{M}_{0}$ such that $A \triangle B=(A \backslash B) \cup(B \backslash A)$ is $M$-null. If, in addition, $\mathscr{M}_{0}$ is countably generated, then we say that $\mathscr{M}$ is $M$ essentially countably generated.

LemMa 3. Let $M: \mathscr{M} \rightarrow X$ be a multimeasure. If $\mathscr{M}$ is $M$-essentially countably generated and if $M$ is non-atomic, then every $m \in \mathscr{S}(M)$ is non-atomic.

Proof. Let $\mathscr{H}_{0}$ be a countably generated $\sigma$-algebra of subsets of $S$ such that $\mathscr{H}_{0} \subset \mathscr{M}$ and $\mathscr{M}$ is $M$-essentially equal to $\mathscr{M}_{0}$. Let $m \in \mathscr{S}(M)$ and suppose that $m$ possesses an atom $A \in \mathscr{M}$. Then there exists $B \in \mathscr{M}_{0}$ such that $A \triangle B$ is $M$-null. It follows that $B$ is an atom of $m_{0}$, the restriction of $m$ to $\mathscr{M}_{0}$. Hence, by Kluvánek (1973), Lemma 1, there exists an atom $D$ of $\mathscr{H}_{0}$ such that $D \subset B$ and $m(D)=m(B)$. Thus $M(D) \neq\{0\}$ and it is easily verified that $D$ is an atom of $M$. This completes the proof.

Lemma 4. Let $X, Y$ be locally convex spaces. Let $M: \mathscr{M} \rightarrow X$ be a perfect multimeasure and $\varphi: X \rightarrow Y$ a continuous linear map. Suppose that $\mathscr{M}$ is $M$-essentially countably generated and $M$ is non-atomic. Then the multimeasure $\varphi \circ M: \mathscr{M} \rightarrow Y$ is non-atomic.

Proof. Certainly $\varphi \circ M$ is a multimeasure from $\mathscr{H}$ to $Y$. Moreover, for every $A \in \mathscr{M}$, we have

$$
(\varphi \circ M)(A)=\{(\varphi \circ m)(A): m \in \mathscr{S}(M)\} .
$$

By Lemma 3, each measure $m \in \mathscr{S}(M)$ is non-atomic. Hence each measure $\varphi \circ m$ is non-atomic by Kluvánek (1973), Lemma 2 . Therefore $\varphi \circ M$ is non-atomic by 
Lemma 2. This completes the proof.

Now let $m: \mathscr{M} \rightarrow X$ be a measure. For any $A \in \mathscr{M}$, we write

$$
R(m, A)=\{m(A \cap B): B \in \mathscr{M}\} .
$$

Let $I$ be an index set directed by the relation $\leqslant . A$ net $\left\{B_{i}: i \in I\right\}$ of sets in $\mathscr{M}$ is said to be $m$-Cauchy (respectively $m$-convergent to $B \in \mathscr{M}$ ) if for every neighbourhood $U$ of 0 in $X$, there exists $i_{0} \in I$ such that $R\left(m, B_{i} \triangle B_{j}\right) \subset U$ for every $i, j \in I$ with $i_{0} \leqslant i$ and $i_{0} \leqslant j$ (respectively $R\left(m, B_{i} \triangle B\right) \subset U$ for every $i \in I$ with $i_{0} \leqslant i$ ).

We say that a measure $m: \mathscr{M} \rightarrow X$ is closed if $\mathscr{M}$ is $m$-complete, that is, every $m$-Cauchy net of sets in $\mathscr{M}$ is $m$-convergent to an element of $\mathscr{M}$. It is known that if $X$ is metrizable, then every measure from $\mathscr{M}$ to $X$ is closed (Kluvánek (1973), Lemma 9).

We close this section by recalling the notion of integral of a function with respect to a vector measure. Let $m: \mathscr{M} \rightarrow X$ be a measure. A measurable real-valued function $f$ on $S$ is said to be $m$-scalarly integrable if it is integrable with respect to every measure $x^{\prime} \circ m, x^{\prime} \in X^{\prime}$. Then, for any $E \in \mathscr{M}$, we denote by $\int_{E} f d m$ the linear form on $X^{\prime}$ defined by

$$
\left\langle x^{\prime}, \int_{E} f d m\right\rangle=\int_{E} f d\left(x^{\prime} \circ m\right),
$$

for every $x^{\prime} \in X^{\prime}$. The function $f$ is said to be $m$-integrable if it is $m$-scalarly integrable and $\int_{E} f d m \in X$ for every $E \in \mathscr{M}$. According to Kluvánek and Knowles (1975), Lemma 1 , p. 26, if the space $X$ is sequentially complete, then every bounded measurable real-valued function on $S$ is $m$-integrable for any measure $m: \mathscr{M} \rightarrow X$.

An $m$-scalarly integrable function $f$ is said to be $m$-null on $E$ if $\int_{A} f d m=0$ for every $A \subset E, A \in \mathscr{K}$.

\section{The range of a perfect multimeasure}

Let $\mathscr{H}$ be a family of measures from $\mathscr{M}$ to $X$. We denote by $\mathscr{N}(\mathscr{H})$ the collection of all sets $E \in \mathscr{H}$ that are $m$-null for every $m \in \mathscr{H}$. For a single measure $m$, we write $\mathscr{N}(m)$ instead of $\mathscr{N}(\{m\})$. Thus

$$
\mathscr{N}(\mathscr{H})=\bigcap\{\mathscr{N}(m): m \in \mathscr{H}\} .
$$

Now let $\mathscr{H}$ be as above and let $E \in \mathscr{M}$. We define $\mathscr{F}(\mathscr{H}, E)$ to be the set of all real-valued bounded measurable functions $f$ on $S$ such that $\int_{E} f d m=0$ for every $m \in \mathscr{H}$ and $f$ is not $m$-null on $E$ if $E \notin \mathscr{N}(m)$. 
Definition 3. A family $\mathscr{H}$ of measures from $\mathscr{H}$ to $X$ is called thin (that is, $\mathscr{K}$ thin) if the set $\mathscr{F}(\mathscr{H}, E)$ is non-empty for every $E \in \mathscr{M} \backslash \mathscr{N}(\mathscr{H})$. A multimeasure $M: \mathscr{M} \rightarrow X$ is said to be thin if $\mathscr{S}(M)$ contains a thin family $\mathscr{H}$ that fills out $M$.

Thus, any thin multimeasure is perfect. If $X=\mathbf{R}^{\infty}$ and if $M$ is a point-valued measure, $M=\left(\mu_{n}\right)$, where the measures $\mu_{n}$ possess a control measure $\nu$ with corresponding density functions $f_{n}$, then $M$ is thin if and only if the set $\left\{f_{n}: n=1,2, \ldots\right\}$ is thin in the sense of Kingman and Robertson (1968), p. 348. In particular, if $X=\mathbf{R}^{n}$ and if $\nu$ is non-atomic, then $M$ is thin (Kingman and Robertson (1968), Lemma 2).

The following example shows that there are thin multimeasures other than pointvalued measures.

EXAmpLe 1. Let $T=[0,1]$ and let $\mathscr{U}$ be a $\sigma$-algebra of subsets of $T$. Also let $v$ be a finite positive measure on $\mathscr{U}$ and define $\mathscr{H}_{0}$ to be the set of all non-negative measures $\mu$ on $\mathscr{U}$ such that $\mu \leqslant \nu$. It is easily verified that for every sequence of measures $\mu_{i} \in \mathscr{H}_{0}(i=1,2, \ldots)$ and every sequence of non-negative $\mathscr{U}$-measurable functions $f_{i}$ on $T(i=1,2, \ldots)$ with $\sum_{i=1}^{\infty} f_{i}(t) \leqslant 1$ for each $t \in T$, there exists $\mu \in \mathscr{H}_{0}$ such that

$$
\sum_{i=1}^{\infty} \int_{I} f_{i} d \mu_{i}=\int_{T}\left(\sum_{i=1}^{\infty} f_{i}\right) d \mu
$$

Now let $Y=[0,1], \mathscr{B}$ the $\sigma$-algebra of Borel sets of $Y$ and $\lambda$ the Lebesgue measure on $\mathscr{B}$. Then let $S=T \times Y$ with the $\sigma$-algebra $\mathscr{H}=\mathscr{U} \otimes \mathscr{B}$. For each $\mu \in \mathscr{H}$, we define a measure $m_{\mu}: \mathscr{M} \rightarrow \mathbf{R}$ as follows

$$
m_{\mu}(A)=\iint_{A} d \lambda d \mu=\int_{T} \lambda\left(A^{\imath}\right) d \mu(t) \quad(A \in \mathscr{K}),
$$

where $A^{l}$ denotes the set of all $y \in Y$ such that $(t, y) \in A$. We prove that the family $\mathscr{H}=\left\{m_{\mu}: \mu \in \mathscr{H}_{0}\right\}$ satisfies the conditions of Lemma 1 . In fact, let $\left(\mu_{i}\right), i=1,2, \ldots$, be a sequence of members of $\mathscr{H}_{0}$ and let $\left(A_{i}\right), i=1,2, \ldots$, be a sequence of disjoint sets in $\mathscr{M}$ with $\bigcup_{i=1}^{\infty} A_{i}=A$. Then, by the property of $\mathscr{H}_{0}$ mentioned above, there exists $\mu \in \mathscr{H}_{0}$ such that

$$
\sum_{i=1}^{\infty} \int_{T} \lambda\left(A_{i}^{t}\right) d \mu_{i}(t)=\int_{T}\left(\sum_{i=1}^{\infty} \lambda\left(A_{i}^{t}\right)\right) d \mu(t)=\int_{T} \lambda\left(A^{t}\right) d \mu(t) .
$$

That is, $\sum_{i=1}^{\infty} m_{\mu_{i}}\left(A_{i}\right)=m_{\mu}(A)$. Therefore, by Lemma 1, the set-valued map $M: \mathscr{M} \rightarrow \mathbf{R}$ defined by $M(A)=\{m(A): m \in \mathscr{H}\}$ is a perfect multimeasure. 
We now verify that $M$ is a thin multimeasure. Let $E \in \mathscr{M} \mid \mathcal{N}(\mathscr{H})$; we define a function $f: S \rightarrow \mathbf{R}$ as follows

$$
\begin{array}{ll}
f(t, y)=y-\left(\lambda\left(E^{\prime}\right)\right)^{-1} \int_{E^{i}} u d \lambda(u) & \text { if } \quad(t, y) \in E \text { and } \lambda\left(E^{\prime}\right) \neq 0, \\
f(t, y)=0 & \text { otherwise. }
\end{array}
$$

Then the function $f$ is bounded, $\mathscr{H}$-measurable and, for every $t \in T$,

$$
\int_{E^{t}} f(t, y) d \lambda(y)=0
$$

It follows that, for every $\mu \in \mathscr{H}_{0}$,

$$
\iint_{E} f d m_{\mu}=\int_{T} \int_{E^{t}} f(t, y) d \lambda(y) d \mu(t)=0 .
$$

It can also be seen that for every $m \in \mathscr{H}$, the function $f$ is not $m$-null on $E$ if $E \notin \mathscr{N}(m)$. Thus, the family $\mathscr{H}$ is $(\mathscr{M}$-)thin and therefore $M$ is a thin multimeasure.

Note that in Example 1 the set-valued map $M_{0}: \mathscr{U} \rightarrow \mathbf{R}$ defined by

$$
M_{0}(A)=\left\{\mu(A): \mu \in \mathscr{H}_{0}\right\},
$$

$A \in \mathscr{U}$, is a perfect multimeasure. Moreover, if $\mathscr{U}$ is embedded in $\mathscr{M}$ by the injection $A \rightarrow A \times Y$, then $M_{0}$ is exactly the restriction of $M$ to $\mathscr{U}$.

More generally we have the following theorem whose proof is similar to the argument presented in Example 1.

Theorem 1. Suppose that $X$ is sequentially complete. Let $M: \mathscr{M} \rightarrow X$ be a multimeasure such that $\mathscr{S}(M)$ contains a family $\mathscr{H}$ that fills out $M$ and has the property that for every sequence $\left(m_{i}\right)$ of members of $\mathscr{H}$ and every sequence $\left(f_{i}\right)$ of nonnegative measurable functions on $S$ with $\sum_{i=1}^{\infty} f_{i} \leqslant 1$, there is an $m \in \mathscr{H}$ satisfying

$$
\sum_{i=1}^{\infty} \int f_{i} d m_{i}=\int\left(\sum_{i=1}^{\infty} f_{i}\right) d m
$$

Then there exist a space $S_{1}$, a $\sigma$-algebra $\mathscr{M}_{1}$ of subsets of $S_{1}$ and a thin multimeasure $M_{1}: \mathscr{M}_{1} \rightarrow X$ such that $\mathscr{M}$ can be embedded in $\mathscr{M}_{1}$ in such a way that $M$ is the restriction of $M_{1}$ to $\mathscr{M}$.

REMARK 1. It is reasonable to ask if there is any relationship between the thinness of $\mathscr{S}(M)$ and the perfectness of $M$. But to our knowledge, this question remains open. We were unable to construct a non-perfect multimeasure $M$ for which $\mathscr{S}(M)$ is thin; the problem being our inability to determine all members of $\mathscr{S}(M)$. We have confined ourselves, in this paper, to the study of perfect multimeasures whose use is common in mathematical economics and control theory. 
LEMma 5. Let $M: \mathscr{H} \rightarrow X$ be a thin multimeasure and let $A \in \mathscr{H}$. Then the setvalued map $M_{A}: \mathscr{M} \rightarrow X$ defined by $M_{A}(B)=M(A \cap B), B \in \mathscr{M}$, is a thin multimeasure.

Proof. Certainly $M_{A}$ is a multimeasure. Suppose that $\mathscr{S}(M)$ contains a thin family $\mathscr{H}$ that fills out $M$. For each $m \in \mathscr{H}$, we define the measure $m_{A}$ by $m_{A}(B)=m(A \cap B), B \in \mathscr{M}$. Then let $\mathscr{H}_{A}=\left\{m_{A}: m \in \mathscr{H}\right\}$. It can be shown easily that the family $\mathscr{H}_{A}$ is thin and fills out $M_{A}$. This completes the proof.

Following Kluvánek and Knowles (1975), p. 82, we call a measure $m: \mathscr{H} \rightarrow X$ a Lyapunov measure if the sets $R(m, A)$ are convex and weakly compact in $X$ for all $A \in \mathscr{K}$. If these sets are merely convex, we will say that $m$ is a convex measure.

Lemma 6. Let $X$ be a Fréchet space and let $\mathscr{H}$ be a thin family of measures from $\mathscr{M}$ to $X$. Then for any $m_{1}, m_{2} \in \mathscr{H}$, the measures $m_{1}$ and $\left(m_{1}, m_{2}\right)$ are Lyapunov.

Proof. Note first that, since $X$ is metrizable, the measures $m_{1}$ and $\left(m_{1}, m_{2}\right)$ are closed. Now let $E \in \mathscr{M} \backslash \mathscr{N}\left(m_{1}\right)$; then $E \in \mathscr{M} \backslash \mathscr{N}(\mathscr{H})$. Hence, by the thinness of $\mathscr{H}$, there is a bounded measurable function $f$ on $S$ such that (i) $\int_{E} f d m=0$ for every $m \in \mathscr{H}$, and (ii) $f$ is not $m$-null on $E$ if $E \notin \mathscr{N}(m)$. Therefore, by Kluvánek and Knowles (1975), Theorem 1, p. 82, the measure $m_{1}$ is Lyapunov.

To prove that the measure $\left(m_{1}, m_{2}\right)$ is Lyapunov, we note that if $E \in \mathscr{H}$ and if $f$ is a bounded measurable function on $S$, then

$$
\int_{E} f d\left(m_{1}, m_{2}\right)=\left(\int_{E} f d m_{1}, \int_{E} f d m_{2}\right) .
$$

Now let $E \in \mathscr{M} \backslash \mathscr{N}\left(\left(m_{1}, m_{2}\right)\right)$. Then either $E \notin \mathscr{N}\left(m_{1}\right)$ or $E \notin \mathscr{N}\left(m_{2}\right)$ and hence $E \notin \mathscr{N}(\mathscr{H})$. Thus, a function $f$ satisfying the conditions (i) and (ii) mentioned above exists and we have

$$
\int_{E} f d\left(m_{1}, m_{2}\right)=0 .
$$

Moreover, since $E \notin \mathscr{N}\left(m_{j}\right)(j=1$ or 2$)$, the function $f$ is not $m_{j}$-null on $E$; hence $f$ is not $\left(m_{1}, m_{2}\right)$-null on $E$. Therefore, again by Kluvánek and Knowles (1975), Theorem 1, p. 82, the measure $\left(m_{1}, m_{2}\right)$ is Lyapunov. This completes the proof.

Lemma 7. Let $M: \mathscr{M} \rightarrow X$ be a perfect multimeasure. Suppose that $\mathscr{S}(M)$ contains a family $\mathscr{H}$ consisting of convex measures such that $\mathscr{H}$ fills out $M$ and for any $m_{1}, m_{2} \in \mathscr{H}$, the measure $\left(m_{1}, m_{2}\right)$ is convex. Then the range of $M$,

is convex.

$$
R(M)=\bigcup\{M(A): A \in \mathscr{M}\},
$$


Proof. Let $x_{1}, x_{2} \in R(M)$ and $0<\lambda<1$. Then there exist $A_{1}, A_{2} \in \mathscr{M}$ and $m_{1}, m_{2} \in \mathscr{H}$ such that $x_{1}=m_{1}\left(A_{1}\right)$ and $x_{2}=m_{2}\left(A_{2}\right)$. Since the measures $m_{1}, m_{2}$ and $\left(m_{1}, m_{2}\right)$ are convex, there exist $B_{1}, B_{2}, B_{3} \in \mathscr{M}$ with $B_{1} \subset A_{1} \mid A_{2}, B_{2} \subset A_{2} \backslash A_{1}$ and $B_{3} \subset A_{1} \cap A_{2}$ such that

$$
\begin{aligned}
m_{1}\left(B_{1}\right) & =\lambda m_{1}\left(A_{1} \backslash A_{2}\right), \\
m_{2}\left(B_{2}\right) & =(1-\lambda) m_{2}\left(A_{2} \backslash A_{1}\right), \\
\left(m_{1}\left(B_{3}\right), m_{2}\left(B_{3}\right)\right) & =\lambda\left(m_{1}\left(A_{1} \cap A_{2}\right), m_{2}\left(A_{1} \cap A_{2}\right)\right) .
\end{aligned}
$$

Let us put $B_{4}=A_{1} \cap A_{2} \backslash B_{3}$ and $B=B_{1} \cup B_{2} \cup B_{3} \cup B_{4}$. We choose any $m_{3} \in \mathscr{S}(M)$ and define the measure $m$ by, for every $A \in \mathscr{M}$,

$$
m(A)=m_{1}\left(A \cap\left(B_{1} \cup B_{3}\right)\right)+m_{2}\left(A \cap\left(B_{2} \cup B_{4}\right)\right)+m_{3}(A \backslash B) .
$$

Then it is clear that $m \in \mathscr{S}(M)$ and $m(B)=\lambda x_{1}+(1-\lambda) x_{2}$. This completes the proof.

THEOREM 2. Let $X$ be a Fréchet space and let $M: \mathscr{M} \rightarrow X$ be a thin multimeasure. Then

(i) $R\left(M_{A}\right)=\bigcup\{M(A \cap B): B \in \mathscr{M}\}$ is convex for every $A \in \mathscr{H}$;

(ii) if for every $A \in \mathscr{H}, M(A)$ contains 0 , then $M(A)$ is convex for all $A \in \mathscr{H}$.

Proof. (i) By Lemma 5, the multimeasure $M_{A}$ is thin. Let $\mathscr{H}_{A}$ be a thin family of measures that fills out $M_{A}$. Then, by Lemma 6 , for any $m_{1}, m_{2} \in \mathscr{H}_{A}$, the measures $m_{1}$ and $\left(m_{1}, m_{2}\right)$ are Lyapunov. Therefore $R\left(M_{A}\right)$ is convex by Lemma 7 . To prove (ii) let $A \in \mathscr{M}$. For any $B \in \mathscr{M}$, since $0 \in M(A \backslash B)$, we have $M(A \cap B) \subset M(A)$. Therefore

$$
M(A)=\bigcup\{M(A \cap B): B \in \mathscr{M}\}=R\left(M_{\mathcal{A}}\right),
$$

which is convex by (i). This completes the proof.

The following lemma generalizes Lemma 5 of Kluvánek (1973).

LEMMA 8. Let $M: \mathscr{K} \rightarrow X$ be a perfect multimeasure. Suppose that $\mathscr{S}(M)$ contains a family $\mathscr{H}$ such that $\mathscr{H}$ fills out $M$ and for every $m \in \mathscr{H}$ and every $x^{\prime} \in X^{\prime}$, the measure $x^{\prime}$ o $m$ is non-atomic. Then the weak closure of $R(M)$ coincides with $\overline{\mathrm{co}} R(M)$ (the closed convex hull of $R(M)$ ).

PRoof. Let $x \in \overline{\operatorname{co}} R(M)$ and let

$$
V=\left\{y \in X:\left|\left\langle y, x_{i}^{\prime}\right\rangle\right|<1, \quad i=1,2, \ldots, n\right\}
$$


be a weak neighbourhood of 0 in $X$. We prove that $x+V$ meets $R(M)$. For each $m \in \mathscr{H}$, define the measure $\mu_{m}: \mathscr{H} \rightarrow \mathbf{R}^{n}$ by

$$
\mu_{m}=\left(x_{1}^{\prime} \circ m, \ldots, x_{n}^{\prime} \circ m\right) \text {. }
$$

Then each $\mu_{m}$ is a non-atomic scalar measure. Let $\mathscr{H}_{0}=\left\{\mu_{m}: m \in \mathscr{H}\right\}$; it is easily verified that the family $\mathscr{H}_{0}$ satisfies the conditions of Lemma 1 . Therefore the set-valued map $M_{0}: \mathscr{M} \rightarrow \mathbf{R}^{n}$ defined by

$$
M_{0}(A)=\left\{\mu(A): \mu \in \mathscr{H}_{0}\right\} \quad(A \in \mathscr{M}),
$$

is a perfect multimeasure. Moreover, since each measure $\mu \in \mathscr{H}_{0}$ is non-atomic (hence convex), it follows from Lemma 7 that the set $R\left(M_{0}\right)=\bigcup\left\{M_{0}(A): A \in \mathscr{M}\right\}$ is convex.

Now since $x \in \overline{\mathrm{co}} R(M)$, there exists $y \in \operatorname{co} R(M)$ such that $y-x \in V$. Let $y=\sum_{j=1}^{p} \lambda_{j} m_{j}\left(A_{j}\right)$, where $m_{j} \in \mathscr{H}, A_{j} \in \mathscr{K}, 0 \leqslant \lambda_{j} \leqslant 1$ for $j=1,2, \ldots, p$ and $\sum_{j=1}^{p} \lambda_{j}=1$. Since for every $j=1,2, \ldots, p, \mu_{m_{j}}\left(A_{j}\right) \in R\left(M_{0}\right)$ which is convex, there exist $m \in \mathscr{H}$ and $A \in \mathscr{M}$ such that

$$
\mu_{m}(A)=\sum_{j=1}^{p} \lambda_{j} \mu_{m_{j}}\left(A_{j}\right)
$$

That is, for every $i=1,2, \ldots, n$,

$$
\begin{aligned}
\left\langle x_{i}^{\prime}, m(A)\right\rangle & =\sum_{j=1}^{p} \lambda_{j}\left\langle x_{i}^{\prime}, m_{j}\left(A_{j}\right)\right\rangle \\
& =\left\langle x_{i}^{\prime}, \sum_{j=1}^{p} \lambda_{j} m_{j}\left(A_{j}\right)\right\rangle \\
& =\left\langle x_{i}^{\prime}, y\right\rangle .
\end{aligned}
$$

Hence, for every $i=1,2, \ldots, n$,

$$
\left|\left\langle x_{i}^{\prime}, m(A)-x\right\rangle\right|=\left|\left\langle x_{i}^{\prime}, y-x\right\rangle\right|<1 .
$$

This shows that $m(A) \in x+V$ and completes the proof.

Remark 2. The result of Lemma 8 had been proved by Tolstonogov (1975), Theorem 6, under different conditions. In fact, Tolstonogov assumed that for each $A \in \mathscr{H}$ there exists a weakly compact set $Q$ in $X$ such that $M(A \cap B) \subset Q$ for every $B \in \mathscr{M}$. In the following example we shall see that a multimeasure may satisfy the conditions of Lemma 8 and yet it may not have the property required by Tolstonogov. 
EXAMPLe 2. Let $(S, \mathscr{M})$ be a measurable space and let $\Lambda$ be a family of finite non-negative non-atomic measures on $\mathscr{M}$. Let $\mathscr{B}$ be the linear space of all realvalued bounded $\mathscr{K}$-measurable functions on $S$. For two functions $f, g \in \mathscr{B}$, we say that they are $\Lambda$-equivalent if $\int|f-g| d \lambda=0$ for every $\lambda \in \Lambda$. Then let $X=\mathscr{B}(\Lambda)$ be the linear space of equivalence classes $\bar{f}, f \in \mathscr{B}$. We furnish $X$ with the locally convex topology $\tau(\Lambda)$ defined by the semi-norms $p_{\lambda}(\bar{f})=\int|f| d \lambda, \lambda \in \Lambda$.

Also, let $\mathscr{B}_{I}(\Lambda)$ denote the subset of $X$ consisting of classes $\bar{f}$ with $0 \leqslant f \leqslant 1$, and let $\mathscr{K}(\Lambda)$ be the subset of $\mathscr{B}_{I}(\Lambda)$ consisting of classes $\bar{\chi}_{A}$, where $\chi_{A}$ is the characteristic function of $A$, for $A \in \mathscr{M}$.

We can choose the family $\Lambda$ so that the set $\mathscr{K}(\Lambda)$ is not $\tau(\Lambda)$-complete (for example, choose $\Lambda$ to be the family of measures equivalent to a non-closed nonatomic vector measure; see Kluvánek and Knowles (1975), pp. 72 and 77, Example 2), and therefore $\mathscr{B}_{I}(\Lambda)$ is not $\tau(\Lambda)$-complete.

Now for each $\bar{f} \in \mathscr{B}_{I}(\Lambda)$, define a map $m_{f}: \mathscr{M} \rightarrow X$ by $m_{f}(A)=\overline{\chi_{A} f}$, for every $A \in \mathscr{K}$. It is easily shown that each $m_{f}$ is a measure and that the family $\mathscr{H}=\left\{m_{f}: \tilde{f} \in \mathscr{B}_{I}(\Lambda)\right\}$ satisfies the conditions of Lemma 1 . Therefore the set-valued map $M: \mathscr{H} \rightarrow X$ defined by $M(A)=\{m(A): m \in \mathscr{H}\}$ is a perfect multimeasure.

Moreover, for each $x^{\prime} \in X^{\prime}$ and each $m_{f} \in \mathscr{H}$, we prove that the measure $x^{\prime} \circ m_{f}$ is non-atomic. In fact, since the linear form $x^{\prime}$ is $\tau(\Lambda)$-continuous, there exist a $\lambda \in \Lambda$, a number $k \geqslant 0$ and a real-valued measure $\mu$ on $\mathscr{H}$ such that $|\mu| \leqslant k \lambda$ and $\left\langle x^{\prime}, \bar{g}\right\rangle=\int g d \mu$ for every $\bar{g} \in X$ (Kluvánek and Knowles (1975), Theorem 1, p. 41). It follows that for each $A \in \mathscr{M}$,

$$
\left(x^{\prime} \circ m_{f}\right)(A)=\left\langle x^{\prime}, \overline{\chi_{A} f}\right\rangle=\int_{A} f d \mu .
$$

Thus $\left|x^{\prime} \circ m_{f}\right| \leqslant k \lambda$ and therefore the non-atomicity of $x^{\prime} \circ m_{f}$ follows from that of $\lambda$.

Finally, we note that the range of the multimeasure $M$ is the whole set $\mathscr{B}_{I}(\Lambda)$ which is convex but not $\tau(\Lambda)$-complete and therefore not weakly compact.

THEOREM 3. Let $M: \mathscr{M} \rightarrow X$ be a perfect multimeasure. If $\mathscr{M}$ is $M$-essentially countably generated and if $M$ is non-atomic, then the weak closure of $R(M)$ coincides with $\overline{\mathrm{co}} R(M)$.

Proof. This follows immediately from Lemmas 3, 4 and 8.

\section{References}

A. Costé (1975), 'Sur les multimesures à valeurs fermées bornées d'un espace de Banach', C. R. Acad. Sci. Paris 280, Série A, 567-570.

A. Costé (1976), 'Densité des sélecteurs d'une multimesure à valeurs convexes fermées bornées d'un espace de Banach séparable', C. R. Acad. Sci. Paris 282, Série A, 967-969. 
J. F. C. Kingman and A. P. Robertson (1968), 'On a theorem of Lyapunov', J. London Math. Soc. 43, 347-351.

I. Kluvánek (1973), 'The range of a vector-valued measure', Math. Systems Theory 7, 44-54.

I. Kluvánek and G. Knowles (1975), Vector measures and control systems (North-Holland, Amsterdam, 1975).

G. Knowles (1975), 'Lyapunov vector measures', Siam J. Control 13, 294-303.

A. Liapounoff (1940), 'Sur les fonctions-vecteurs complètement additives', Bull. Acad. Sci. URSS Sér. Math. (Izvestiya Akad. Nauk SSSR Ser. Mat.) 4, 465-478.

A. A. Tolstonogov (1975), 'On the theorems of Radon-Nikodym and A. A. Lyapunov for a multivalued measure', Soviet Math. Dokl. 16, 1588-1592 (Dokl. Akad. Nauk SSSR, 225, 1023-1026).

Department of Mathematics

University of Western Australia

Nedlands 6009

Australia 